\title{
Pediatric Medullary Stroke, Severe Dysphagia, and Multimodal Intervention
}

\author{
Laura Brooks $^{1}$ (D) Nikhila Raol $^{1,2} \cdot$ Steven Goudy ${ }^{1,2} \cdot$ Caroline Ivie $^{1}$
}

Received: 30 November 2020 / Accepted: 12 February 2021 / Published online: 27 October 2021

(c) The Author(s), under exclusive licence to Springer Science+Business Media, LLC, part of Springer Nature 2021

\begin{abstract}
Lateral medullary syndrome/Wallenberg syndrome is a stroke in the lateral medulla with symptoms often including dysphagia and dysphonia. In adults, this stroke is the most common brainstem stroke, but it is rare in the pediatric population. Insults to the medulla can involve the "swallowing centers," the nucleus ambiguus and nucleus tractus solitarius, and the cranial nerves involved in swallowing, namely IX (glossopharyngeal) and X (vagus). These individuals can develop severe dysphagia with an inability to trigger a swallow due to pharyngeal weakness and impaired mechanical opening of the upper esophageal sphincter (UES) which can result in aspiration. We present a 7-year-old male with 22q11.2 deletion syndrome (velocardiofacial syndrome) and velopharyngeal insufficiency who underwent pharyngeal flap surgery at an outside hospital whose post-operative course was complicated by adenovirus, viral myocarditis, and dorsal medullary stroke. He required a tracheostomy and gastrostomy tube. He was discharged from that hospital and readmitted to our hospital 4 months later for increased oxygen requirement, requiring a 5 month admission in the intensive care units. His initial VFSS revealed absent UES opening with the entire bolus remaining in the pyriform sinuses resulting in aspiration. His workup over the course of his admission included multiple videofluoroscopic swallow studies (VFSS), flexible endoscopic evaluation of swallowing (FEES), and pharyngeal and esophageal manometry. Intervention included intensive speech therapy, cricopharyngeal Botox ${ }^{\circledR}$ injection, and cricopharyngeal myotomy. Nineteen months after his stroke, he transitioned to oral intake of solids and liquids with adequate movement of the bolus through the pharynx and UES and no aspiration on his VFSS.
\end{abstract}

Keywords Pediatric dysphagia $\cdot$ Medullary stroke $\cdot$ Cricopharyngeal myotomy $\cdot$ Pharyngeal and esophageal manometry

\section{Clinical Conundrum}

Our patient who was born with 22q11.2 deletion syndrome (velocardiofacial syndrome) with a submucous cleft palate and velopharyngeal incompetence underwent a pharyngeal flap surgery at an outside hospital. Following the surgery, he developed postoperative viral myocarditis and respiratory failure, requiring a tracheostomy and gastrostomy tube placement. An MRI of the brain showed hemorrhagic stroke in the dorsal medulla and scattered tiny foci of microhemorrhage in the cerebellar hemisphere. He was admitted

Laura Brooks

laura.brooks@choa.org

1 Children's Healthcare of Atlanta, 1405 Clifton Road NE, Atlanta, GA 30322, USA

2 Department of Otolaryngology-Head and Neck Surgery, Emory University School of Medicine, Atlanta, GA 30322, USA to our hospital 4 months after discharge from the outside hospital for increased secretions and increased respiratory requirements requiring ventilator support. He presented with dysphonia, dysarthria, facial asymmetry, and inability to swallow his secretions requiring frequent oral and tracheal suctioning. He had been NPO since the surgery/ stroke per parent report ( 8 months) and was referred for an esophagram and videofluoroscopic swallow study (VFSS). The esophagram results were "limited by minimal intake." The VFSS immediately followed, and he presented with significant oral, pharyngeal, and esophageal deficits most saliently characterized by severely impaired upper esophageal sphincter (UES) opening resulting in no movement of the bolus into the esophagus (Figs. 1,2). The material eventually was aspirated without a cough response, despite his efforts to swallow. A flexible endoscopic evaluation of swallowing (FEES) was performed, and the patient required $11 \mathrm{~min}$ of extensive oral, nasal, and pharyngeal suction to clear the excessive pooling secretions (because he could not swallow) 


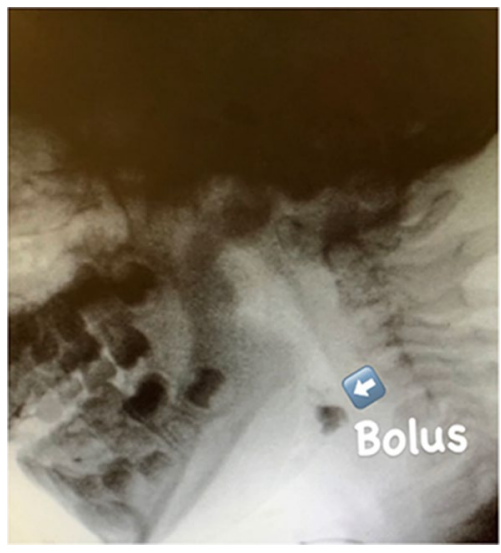

Fig. 1 First view of VFSS after esophagram, material still present on UES approximately $20 \mathrm{~min}$ later

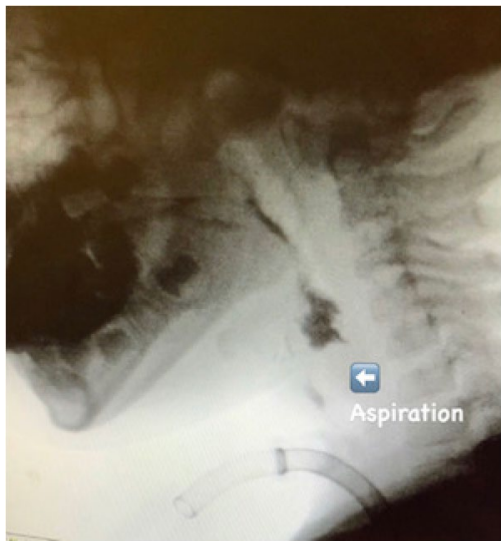

Fig. 2 No movement of bolus into esophagus despite strategies. Aspiration

for adequate viewing of the pharynx and larynx. Following the extensive suctioning, his larynx was visualized via oral presentation of the flexible scope after attempts via nasal passage were not effective. The true vocal folds were observed, and he could adequately adduct both vocal cords, with partial abduction (left arytenoid movement greater than right) (Figs. 3, 4). Food and drink presentations were deferred due to oral scope presentation and patient fatigue/ agitation. Two weeks later, esophagogastroduodenoscopy was performed, with the scope passing easily into the esophagus through the UES.

\section{What is the Etiology of His Severe Dysphagia?}

Our patient's presentation is most consistent with a lateral medullary infarct or Wallenberg syndrome, with symptoms often including dysphagia with UES dysfunction and dysphonia [1]. First procedure: He underwent Botox ${ }^{\circledR}$ injection of the cricopharyngeus muscle given the temporary

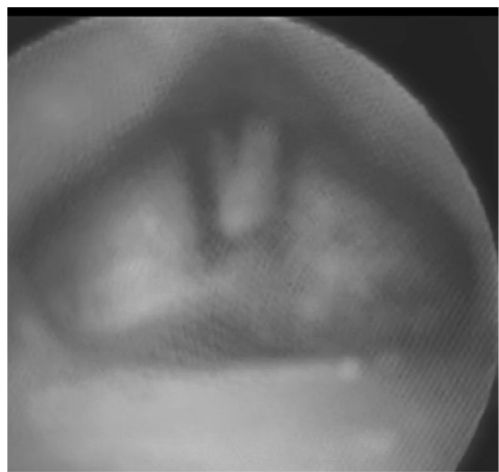

Fig. 3 Vocal cords adducted during the FEES

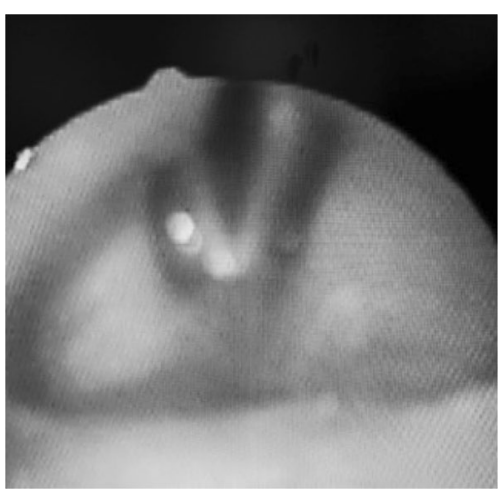

Fig. 4 Vocal cords abducted during the FEES. Of note, Passy Muir Valve transtracheal pressure was measured via manometry and was $<10 \mathrm{~cm} \mathrm{H}_{2} \mathrm{O}$, demonstrating adequate ability to exhale through his nose and mouth

effects of this procedure and success with some UES disorders. Second VFSS following Botox ${ }^{\circledR}$ : Anterior-posterior view revealed that the bolus remained exclusively in the right pyriform sinus, so a right head turn was cued to direct subsequent bolus presentations to the presumed stronger side of the pharynx (left). A trace amount of barium was noted to enter the esophagus (Fig. 5). Intensive speech therapy followed and included small PO trials of thin liquids with sensory input (flavor and temperature), cueing right head turn, prompting effortful swallows, and dry swallows. Cough was addressed with Passy Muir Valve and expiratory muscle strength training (EMST). Mendelsohn maneuver and Shaker exercises were not taught due to patient's age and reports of discomfort. Second Procedure: He underwent endoscopic cricopharyngeal myotomy, submandibular gland excision, and parotid duct ligation. Third VFSS following cricopharyngeal myotomy: The patient demonstrated movement of part of the bolus from the pharynx through the upper esophageal sphincter $\times 5$ with strategies (cold temperature, head tilt to left, head turn to right, effortful swallow). Some residue remained 


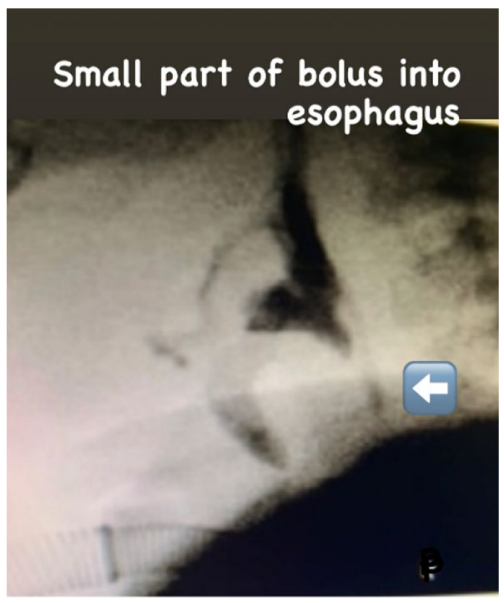

Fig. 5 VFSS after botox injection to cricopharyngeus muscle

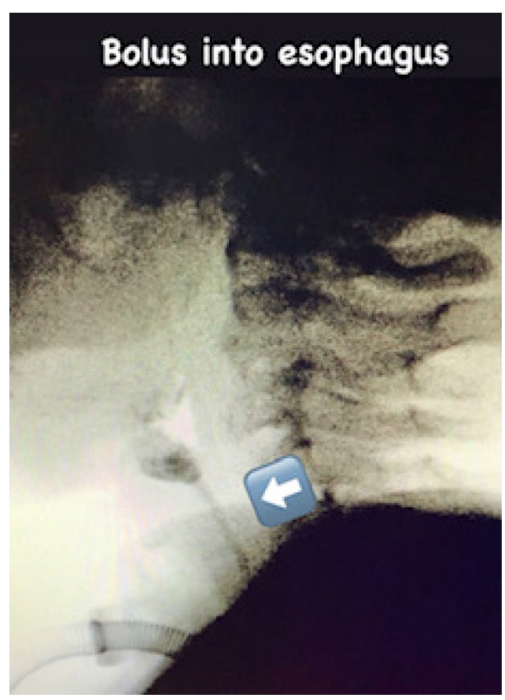

Fig. 6 After CP myotomy

in his pyriform sinuses which were silently aspirated (Fig. 6). Third procedure: Pharyngeal and esophageal manometry were performed with the probe in right nostril (note patient with pharyngeal flap), and results revealed UES: normal UES relaxation (after myotomy), good bolus transit through UES but very poor to no pharyngeal contractions. Fourth VFSS prior to discharge: The patient was positioned both in upright and sidelying on his left side (presumed stronger side) resulting in improvement in swallow function demonstrating more efficient movement of the thin liquid bolus through the UES (Fig. 7). Fifth VFSS (outpatient): The patient consumed solids and thin liquids, and the boluses moved adequately through the pharynx, UES, and esophagus with left head tilt (his preferred strategy). Mild residue in the pyriform sinuses

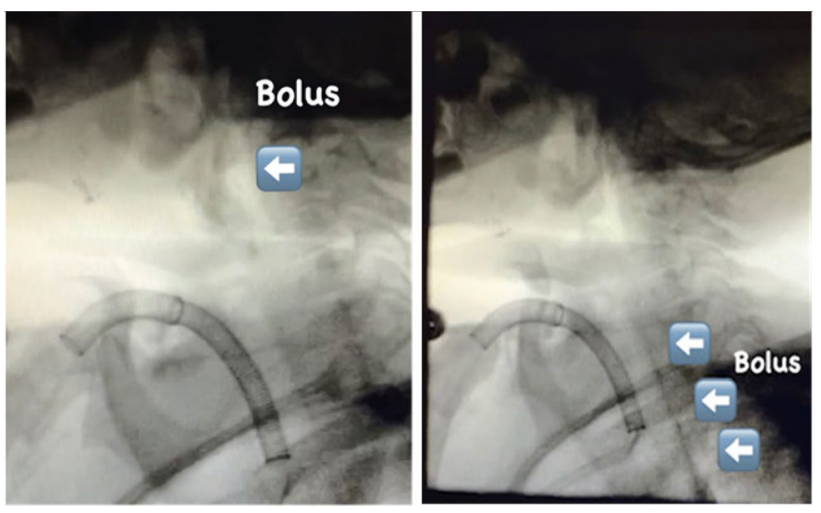

Fig. 7 VFSS swallow sequence of thin liquids with strategies prior to discharge from hospital
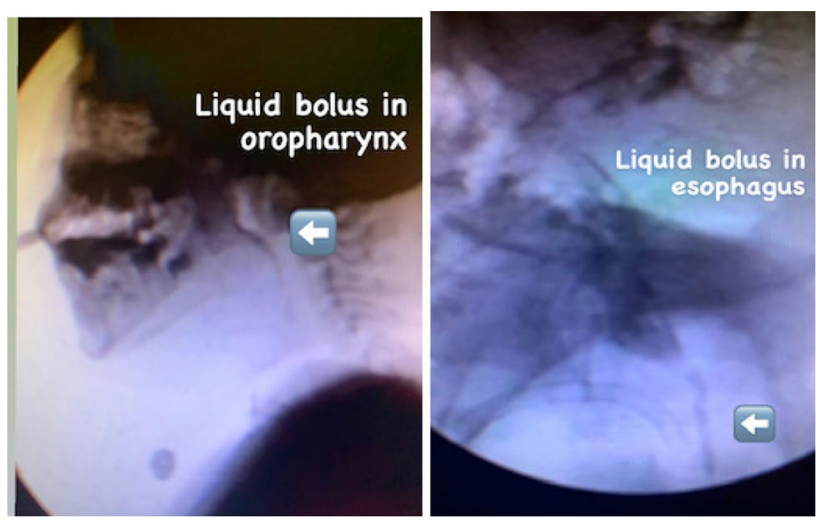

Fig. 8 Final VFSS thin liquids-head tilt

cleared with subsequent swallows, with no laryngeal penetration or aspiration events occurred with any presentations (Figs. 8, 9).

\section{Discussion}

Swallowing involves bilateral input from the swallowing centers in the rostral dorsolateral medulla, and the major swallowing centers include the nucleus tractus solitarius, nucleus ambiguus, and the reticular formation $[2,3]$. Our pediatric patient presented with severe dysphagia after a dorsal medullary stroke, and his presentation was most consistent with lateral medullary infarct, or Wallenberg syndrome given his dysphonia and severe dysphagia characterized by inability to trigger a swallow, unilateral pharyngeal weakness, decreased pressure on the bolus, and little to no opening of the UES resulting in aspiration [1, 4]. A Botox ${ }^{\circledR}$ injection into the cricopharyngeus muscle was minimally effective for this patient. This information paired with relaxed UES during sedated endoscopy ruled 
Fig. 9 Final VFSS yogurthead tilt
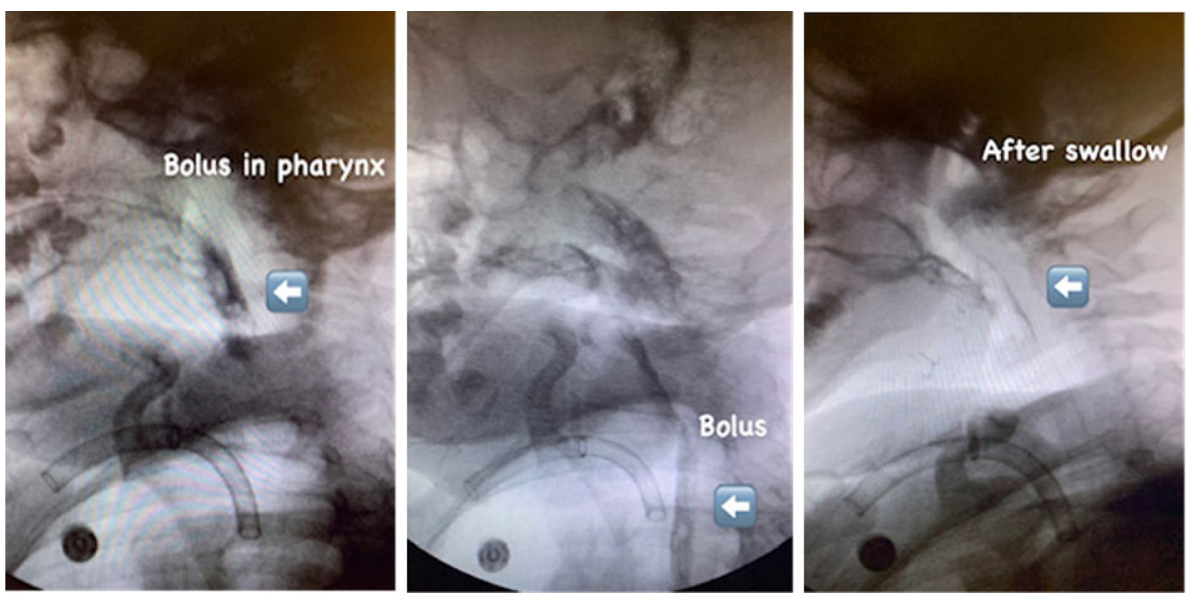

out hypertonicity as an etiology of UES dysfunction and pointed to possible impaired mechanical opening of the UES, as seen in patients with lateral medullary infarct. Our patient underwent instrumental swallow studies, intensive swallowing therapy, pharyngeal/esophageal manometry, and a cricopharyngeal myotomy given the severity of his dysphagia 10 months post-stroke [4]. His final VFSS revealed adequate movement of thin liquids and solids through the pharynx and UES without aspiration, and he has transitioned to an oral diet using his gastrostomy tube for supplemental nutrition as needed.

Limitations of this clinical conundrum include lack of access to the MRI results other than the information presented in this paper as it was performed at an outside hospital. The results communicated to our hospital did not include the words "lateral" or reference to "left" versus "right" side.

Acknowledgements Nikhila Raol is supported by the American Society of Pediatric Otolaryngology Career Development Award. There is no conflict of interest pertaining to the award.

\section{Declarations}

Conflict of interest The authors declare that they have no conflict of interest. There are no financial disclosures associated with this paper.

Informed Consent Informed consent was obtained for the participant in this report.

\section{References}

1. Sheffler K. Lateral medullary syndrome (Wallenberg syndrome) and dysphagia: an analysis of the literature and case studies. (n.d.). https://swallowstudy.com/wp-content/uploads/LMS-WallenbergSyndrome-Literaturereview-and-case-studies.pdf.

2. Vigderman AM, Chavin JM, Kososky C, Tahmoush AJ. Aphagia due to pharyngeal constrictor paresis from acute lateral medullary infarction. J Neurol Sci. 1998;155(2):208-10.

3. Aydogdu I, Ertekin C, Tarlaci S, Turman B, Kiylioglu N, Secil Y. Dysphagia in lateral medullary infarction (Wallenberg's syndrome): an acute disconnection syndrome in premotor neurons related to swallowing activity? Stroke. 2001;32(9):2081-7.

4. Logemann JA. Evaluation and treatment of swallowing disorders. disorders. 2nd ed. Austin TX: Pro-Ed; 1998. p. 308-9.

Publisher's Note Springer Nature remains neutral with regard to jurisdictional claims in published maps and institutional affiliations.

Laura Brooks MEd CCC-SLP, BCS-S

Nikhila Raol MD, MPH

Steven Goudy MD

Caroline Ivie PA 